\title{
340.
}

\section{A SECOND MEMOIR ON SKEW SURFACES, OTHERWISE SCROLLS.}

[From the Philosophical Transactions of the Royal Society of London, vol. CLIV. (for the year 1864), pp. 559-576. Received April 29,-Read May 26, 1864.]

THE principal object of the present memoir is to establish the different kinds of skew surfaces of the fourth order, or Quartic Scrolls; but, as preliminary thereto, there are some general researches connected with those in my former memoir "On Skew Surfaces, otherwise Scrolls" ${ }^{1}$ ), and I also reproduce the theory (which may be considered as a known one) of cubic scrolls; there are also some concluding remarks which relate to the general theory. As regards quartic scrolls, I remark that M. Chasles, in a footnote to his paper, "Description des courbes de tous les ordres situées sur les surfaces réglées du troisième et du quatrième ordres" $\left({ }^{2}\right)$, states, "les surfaces réglées du quatrième ordre.... admettent quatorze espèces." This does not agree with my results, since I find only eight species of quartic scrolls; the developable surface or "torse" is perhaps included as a "surface réglée;" but as there is only one species of quartic torse, the deficiency is not to be thus accounted for. My enumeration appears to me complete, but it is possible that there are subforms which M. Chasles has reckoned as distinct species.

\section{On the Degeneracy of a Scroll, Article Nos. 1 to 5 .}

1. A scroll considered as arising from any geometrical construction, for instance one of the scrolls $S(m, n, p), S\left(m^{2}, n\right), S\left(m^{3}\right)$ considered in my former memoir, or say in general the scroll $S$, may break up into two or more inferior scrolls $S^{\prime}, S^{\prime \prime}, \ldots$; but as long as $S^{\prime}, S^{\prime \prime}, \ldots$ are proper scrolls (not torses, and $\grave{a}$ fortiori not cones or planes), no one of these can be considered, apart from the others, as the result of the geometrical

1 Philosophical Transactions, vol. cLIIr. (1863), pp. 453-483, [339].

2 Comptes Rendus, t. LIIr. (1861), see p. 888.

c. V. 
construction, and we can only say that the scroll $S$ given by the construction is the aggregate of the scrolls $S^{\prime}, S^{\prime \prime}, \ldots$; and the like when we have the scrolls $S^{\prime}, S^{\prime \prime}, \ldots$, each repeated any number of times, or say when $S=S^{\prime \alpha}{ }^{\prime \prime \prime} \ldots$... Suppose however that the scrolls $S^{\prime \prime}, S^{\prime \prime}, \ldots$ are any one or more of them a torse or torses-or, to make at once the most general supposition, say that we have $S=\Sigma S^{\prime}$, where $\Sigma$ is a torse, or aggregate of torses $\left(\Sigma=\Sigma^{\prime} a \Sigma^{\prime \prime \beta} \ldots\right)$, and $S^{\prime}$ is a proper scroll or aggregate of proper scrolls; then, although it is not obligatory to do so, we may without impropriety throw aside the torse-factor $\Sigma$, and consider the original scroll $S$ as degenerating into the scroll $S^{\prime}$, and as suffering a reduction in order accordingly.

2. As an illustration, consider the scroll $S(m, n, p)$ generated by a line which meets three directrix curves of the orders $m, n, p$ respectively; and assume that the curves $m, n, p$ are each of them situate on the same scroll $\Sigma$, the curve $m$ meeting each generating line of $\Sigma$ in $\alpha$ points, the curve $n$ each generating line in $\beta$ points, and the curve $p$ each generating line in $\gamma$ points. Each generating line of $\Sigma$ is $\alpha \beta \gamma$ times a generating line of $S$, and we have $S=\Sigma^{a \beta \gamma} S^{\prime}$, where $S^{\prime}$ may be a proper scroll ; it is however to be noticed that if the curves $m, n, p$ any two of them intersect, $S^{\prime}$ will itself break up and contain cone-factors, as will presently appear. And if $\Sigma$, instead of being a proper scroll, be a torse, then we may consider $S$ as degenerating into $S^{\prime}$, the reduction in order being of course $=\alpha \beta \gamma \times$ order of $\Sigma$.

3. But this is not the only way in which the scroll $S(m, n, p)$ may degenerate; for suppose that two of the directrix curves, say $n$ and $p$, intersect, then the lines from the point of intersection to the curve $m$ form a cone of the order $m$ which will present itself as a factor of $S$; and generally if the curves $n$ and $p$ intersect in $\alpha$ points, the curves $p$ and $m$ in $\beta$ points, and the curves $m$ and $n$ in $\gamma$ points, then we have $\alpha$ cones each of the order $m, \beta$ cones each of the order $n$, and $\gamma$ cones each of the order $p$, or say $S=C S^{\prime}$, where $C$ is the aggregate of the cone-factors; and the scroll $S$ degenerates into $S^{\prime}$, the reduction in order being $=\alpha m+\beta n+\gamma p$. It is hardly necessary to remark that if a point of intersection of two of the curves is a multiple point on either or each of the curves, it is, in reckoning the number of intersections of the two curves, to be taken account of according to its multiplicity in the ordinary manner.

4. There is yet another case to be considered: suppose that the curves $n$ and $p$ lie on a cone, and that the curve $m$ passes through the vertex of this cone; this cone, repeated a certain number of times, is part of the locus, or we have $S=C^{\theta} S^{\prime}$, so that the scroll $S$ degenerates into $S^{\prime}$, the reduction in order being $=\theta \times$ order of cone. If, to fix the ideas, the curves $n$ and $p$ are respectively the complete intersections of the cone by two surfaces of the orders $g, h$ respectively (this implies $n=g k, p=h k$, if $k$ be the order of the cone), which surfaces do not pass through the vertex of the cone, and if, moreover, the vertex of the cone be an $a$-tuple point on the curve $m$, then $\theta=a g h$, and the reduction in order is $=a g h k$.

5. The foregoing causes of reduction, or some of them, may exist simultaneously; it would require a further examination to see whether the aggregate reduction is in 
all cases the sum of the separate reductions. But the aggregate reduction once ascertained, then writing $S(m, n, p)$ for the order of the reduced scroll, we shall have

$$
S(m, n, p)=2 m n p-\text { Reduction. }
$$

In particular, in the case above referred to, where the curves $n$ and $p, p$ and $m$, $m$ and $n$ meet in $\alpha, \beta, \gamma$ points respectively, but there is no other cause of reduction,

$$
S(m, n, p)=2 m n p-a m-\beta n-\gamma p,
$$

which is a formula which will be made use of.

The foregoing investigations apply, muiatis mutandis, to the scrolls $S\left(m^{2}, n\right), S\left(m^{3}\right)$; but I do not at present enter into the development of them in regard to these scrolls.

\section{Scrolls with two directrix lines, Article Nos. 6 to 11.}

6. Consider now a scroll having two directrix lines: it may be assumed that these do not intersect; for if they did, then any generating line, quà line meeting the two directrix lines, would either lie in the plane of the two lines, or else would pass through their point of intersection; that is, the scroll would break up into the plane of the two lines, considered as the locus of the tangents of a plane curve, and into a cone having for its vertex the point of intersection of the two lines. Each generating line meets any plane section of the scroll in the point where such generating line meets the plane of the section; the plane section constitutes a third directrix; or the scrolls in question are all included in the form $S(1,1, m)$, where $m$ is a plane curve. The order of the scroll $S(1,1, m)$ is in general $=2 m$; but if the one line meets the curve $\alpha$ times, that is, in an $\alpha$-tuple point of the curve, and the other line meets the curve $\beta$ times, that is, in a $\beta$-tuple point of the curve, then by the general formula (ante, No. 5) the order of the scroll is $=2 m-\alpha-\beta$; and in particular if $\alpha+\beta=m$, then the order is $=m$.

7. We may without loss of generality attend only to the last-mentioned case. To show how this is, suppose for a moment that the two lines do not either of them meet the curve; the scroll is then of the order $2 \mathrm{~m}$. Call the point in which each line meets the plane of the curve the foot of this line, then the line joining the two feet meets the curve in $m$ points; and it is in respect of each of these points a generating line of the scroll; that is, it is an $m$-tuple generating line: the section of the scroll by the plane of the curve $m$ is in fact this line counting $m$ times, and the curve $m ; m+m=2 m$, the order of the scroll. And in like manner the section by any plane through the $m$-tuple line is this line counting $m$ times, and a curve of the order $m$ not meeting either of the directrix lines. But the section by any other plane is a curve of the order $2 m$ meeting each of the directrix lines in a point which is an $m$-tuple point of the section (each directrix line is in fact an $m$-tuple line of the scroll); and by considering, in place of the particular section $m$, this general section, we have the scroll of the order $2 m$ in the form $S(1,1,2 m)$, where the two directrix lines each meet the section $m$ times; so that the order is $4 m-m-m=2 m$. 
8. And so in general, $m$ being a plane curve, when the scroll $S(1,1, m)$ is of an order superior to $m$, say $=m+k$, this only means that the section chosen for the directrix curve $m$ is not the complete section by the plane of such curve, but that the line joining the feet of the two directrix lines is a $k$-tuple generating line of the scroll, and that the complete section is made up of this line counting $k$ times and of the curve $m$. So that taking, not the section through the multiple generating line, but the general section, for the plane directrix curve, the only case to be considered is that in which the section is a proper curve of an order equal to that of the scroll; or, what is the same thing, we have only to consider the scrolls $S(1,1, m)$ for which the order is depressed from $2 m$ to $m$ in consequence of the directrix lines meeting the plane section $\alpha$ times and $\beta$ times, that is, in an $\alpha$-tuple point and a $\beta$-tuple point respectively, where $\alpha+\beta=m$.

9. It is clear that in the case in question the directrix lines are an $\alpha$-tuple line and a $\beta$-tuple line respectively. The generation is as follows: Scroll $S(1,1, m)$ of the order $m$; the curve $m$ being a plane curve of the order $m$ having an $\alpha$-tuple point and a $\beta$-tuple point, where $\alpha+\beta=m$ : the directrix lines, say 1 and $1^{\prime}$, pass through these points respectively, and they do not intersect each other. The generating lines pass through the directrix lines 1 and $\mathbf{1}^{\prime}$ and the curve $m$, and we have thence the scroll $S(1,1, m)$. Taking at pleasure any point on the curve $m$, we can through this point draw a single line meeting each of the directrix lines $1,1^{\prime}$; that is, the curve $m$ is a simple curve on the scroll. Taking at pleasure a point on the directrix line 1, and making this the vertex of a cone standing on the curve $m$, this cone has an $\alpha$-tuple line (the line 1 ) and a $\beta$-tuple line (the line joining the vertex with the foot of the line $1^{\prime}$ ); the line $1^{\prime}$ meets this cone in the foot of the line $1^{\prime}$, counting $\beta$ times, and besides in $m-\beta,=\alpha$ points; the lines joining the vertex with the lastmentioned points respectively (or, what is the same thing, the lines, other than the $\beta$-tuple line, in which the plane through the vertex and the line $1^{\prime}$ meets the cone) are the $\alpha$ generating lines through the assumed point on the line 1 ; and the line 1 is thus an $\alpha$-tuple line of the scroll. And in like manner, through an assumed point of the directrix line 1 , we construct $\beta$ generating lines of the scroll; and the line $1^{\prime}$ is a $\beta$-tuple line of the scroll.

10. The scroll $S(1,1, m)$ now in question has not in general any multiple generating line; in fact a multiple generating line would imply a corresponding multiple point on the section $m$; and this section, assumed to be a curve having an $\alpha$-tuple point and a $\beta$-tuple point, has not in general any other multiple point. But it may have other multiple points; and if there is, for example, a $\gamma$-tuple point, then the line from this point which meets the two directrix lines counts $\gamma$ times, or it is a $\gamma$-tuple generating line; and so for all the multiple points of $m$ other than the $\alpha$-tuple point and the $\beta$-tuple point which correspond to the directrix lines respectively. It is to be noticed that the multiplicity $\gamma$ of any such multiple generating line is at most equal to the smallest of the two numbers $\alpha$ and $\beta$; for suppose $\gamma>\alpha$, then, since $\alpha+\beta=m$, we should have $\gamma+\beta>m$, and the line joining the $\gamma$-tuple point and the $\beta$-tuple point would meet the curve $m$ in $\gamma+\beta$ points, which is absurd. In the 
case of several multiple lines, there are other conditions of inequality preventing selfcontradictory results $\left({ }^{1}\right)$.

11. The general section is a curve of the order $m$, having an $\alpha$-tuple point and a $\beta$-tuple point corresponding to the directrix lines respectively, and a $\gamma$-tuple point, \&c.... corresponding to the other multiple points (if any). A section through the directrix line 1 is in general made up of this line, counting $\alpha$ times, and of $\beta$ generating lines passing through one and the same point of the directrix line $1^{\prime}$; if the section pass also through a $\gamma$-tuple generating line, then, of the $\beta$ generating lines in question, $\gamma$ (which, as has been seen, is $\ngtr \beta$ ) unite together in the $\gamma$-tuple generating line; and so for the sections through the directrix line $1^{\prime}$. The general section through a $\gamma$-tuple generating line is this line counting $\gamma$ times, and a curve of the order $m-\gamma$, which has an $(\alpha-\gamma)$ tuple point at its intersection with the directrix line 1 , and $a(\beta-\gamma)$ tuple point at its intersection with the directrix line $1^{\prime}$; it has a $\delta$-tuple point, \&c... at its intersections with the other multiple generating lines, if any.

\section{Scrolls with a twofold directrix line, Article Nos. 12 to 16.}

12. But there is a case included indeed as a limiting one in the foregoing general case, but which must be specially considered; viz. the two directrix lines $\mathbf{1}$ and $\mathbf{1}^{\prime}$ may coincide, giving rise to a twofold directrix line. To show how this is, I return for the moment to the case of the scroll $S(1,1, m)$ with two distinct directrix lines 1 and $1^{\prime}$, and, to fix the ideas, I suppose that the directrix lines do not either of them meet the curve $m$, so that the order of the scroll is $=2 m$. Through the line 1 imagine the series of planes $A, B, C, \ldots$ meeting the line $1^{\prime}$ in the points $a^{\prime}, b^{\prime}, c^{\prime} \ldots$; the generating lines through the point $a^{\prime}$ are the lines in the plane $A$ to the points in which this plane meets the curve $m$; the generating lines through the point $b^{\prime}$ are the lines in the plane $B$ to the points where this plane meets the curve $m$; and so for the generating lines through the points $c^{\prime}, d^{\prime} \ldots$; and it is clear that the points $a^{\prime}, b^{\prime}, c^{\prime}, \ldots$ correspond homographically with the planes $A, B, C, \ldots$ This gives immediately the construction for the case where the two directrix lines come to coincide. In fact, on the twofold directrix line $1=1^{\prime}$ take the series of points $a, b, c \ldots$, and through the same line, corresponding homographically to these points, the series of planes $A, B, C, \ldots$; the generating lines through the point $a$ are the lines through this point, in the plane $A$, to the points in which this plane meets the curve $m$; and so for the entire series of points $b, c, \ldots$ of the line $1=1^{\prime}$; the resulting scroll, which $I$ will designate as the scroll $S(\overline{1,1}, m)$, remains of the order $=2 m$. If there is given a point of the curve $m$, then the plane through this point and the directrix line is the plane $A$; and the point $a$ is then also given by the homographic correspondence of the series of planes and points, and the generating line through the given point on the curve $m$ is the line joining this point with the point $a$.

1 Suppose, for example (see next paragraph of the text), that there were a $\gamma$-tuple generating line and a $\delta$-tuple generating line lying in plano with the line 1 ; these lines counting as $(\gamma+\delta)$ lines, must be included among the $\beta$ generating lines through the plane in question; this implies that $\gamma+\delta \neq \beta$, a conclusion which must be obtainable from consideration of the curve $m$ irrespectively of the scroll. 
13. We may say that, in regard to any point $a$ of the line 1 , the corresponding plane $A$ is the plane of approach of the coincident line $1^{\prime}$; and that in regard to the same point $a$ and to any plane through it, the trace on that plane of the plane of approach is the line of approach of $1^{\prime}$; that is, we may consider that the coincident directrix line $1^{\prime}$ meets the plane through $a$ in a consecutive point on the line of approach. In particular if the point $a$ be the foot of the directrix line 1 (that is, the point where this line meets the plane of the curve $m$ ), and the plane through $a$ be the plane of the curve $m$, then the intersection of the last-mentioned plane by the plane $A$ which corresponds to the point $a$ is the line of approach, and the foot of the coincident directrix line $1^{\prime}$ is the consecutive point to $a$ along the line of approach. The expression "the line of approach," used absolutely, has always the signification just explained, viz. it is the intersection of the plane of the curve $m$ by the plane corresponding to the foot of the directrix line.

14. Suppose now that the line 1 meets the curve $m$, or, more generally, meets it $\alpha$ times, that is, in an $\alpha$-tuple point; it might at first sight appear that the coincident line $1^{\prime}$ should also be considered as meeting the curve $\alpha$ times, and that the resulting scroll should be of the order $2 m-\alpha-\alpha=2 m-2 \alpha$. But this is not the case; so long as the direction of the line of approach is arbitrary, the line $\mathbf{1}^{\prime}$ must be considered as a line indefinitely near to the line 1 , but nevertheless as a line not meeting the curve at all; and the order of the scroll is thus $=2 m-\alpha$. If, however, the line of approach is the tangent to a branch through the $\alpha$-tuple point-that is, if the plane corresponding to the $\alpha$-tuple point meet the plane of the curve in such tangent, then the coincident line $1^{\prime}$ is to be considered as meeting the curve $m$ in a consecutive point on such branch, and the order of the scroll is $=2 m-\alpha-1$. And so if at the multiple point there are $\beta$ branches having a common tangent, then the coincident line $1^{\prime}$ is to be considered as meeting the curve $m$ in a consecutive point along each of such branches, or say in a consecutive $\beta$-tuple point along the branch, and the order of the scroll sinks to $2 m-\alpha-\beta$. The point spoken of as the $\alpha$-tuple point is, it should be observed, more than an $\alpha$-tuple point with a $\beta$-fold tangent; it is really a point of union of an $\alpha$-tuple point and a $\beta$-tuple point, or say a united $\alpha(+\beta)$ tuple point, equivalent to

$$
\frac{1}{2} \alpha(\alpha-1)+\frac{1}{2} \beta(\beta-1)
$$

double points or nodes; and the case is precisely analogous to that of the scroll $S(1,1, m)$, where the two directrix lines pass through an $\alpha$-tuple point and a $\beta$-tuple point of the curve $m$ respectively. It may be added that if at the multiple point in question, besides the $\beta$ branches having a common tangent, there are $\gamma$ branches having a common tangent, then the point is, so to speak, a united $\alpha(+\beta,+\gamma)$ tuple point equivalent to $\frac{1}{2} \alpha(\alpha-1)+\frac{1}{2} \beta(\beta-1)+\frac{1}{2} \gamma(\gamma-1)$ double points or nodes; but the order of the scroll is still $=2 m-\alpha-\beta$.

15. In the same way as the scrolls $S(1,1, m)$ are all included in the case where the order of the scroll, instead of being $=2 m$, is $=m$, so that the scrolls $S(\overline{1,1}, m)$ are all included in the case where the order of the scroll, instead of being $=2 m$, is $=m$. 
That is, we may suppose that the curve $m$ has a united $\alpha(+\beta)$ tuple point $(\alpha+\beta=m)$, and may take the directrix line to pass through this point, and the line of approach to be the common tangent of the $\beta$ branches; and this being so, the order of the scroll will be $2 m-\alpha-\beta,=m$. It may be added that if the curve $m$ has, besides the $\alpha(+\beta)$ tuple point, a $\gamma$-tuple point, then the scroll will have a $\gamma$-tuple generating line, and $s o$ for the other multiple points of the curve $m$.

16. We may, in the same way as for the scroll $S(1,1, m)$, consider the different sections of the scroll $S(\overline{1,1}, m)$ of the order $m$. The general section is a curve of the order $m$, having an $\alpha(+\beta)$ tuple point at the intersection with the directrix line, and a $\gamma$-tuple point, \&c. corresponding to the multiple generating lines, if any. A section through the directrix line is in general made up of this line counting $\alpha$ times, and of $\beta$ generating lines through the point which corresponds to the plane of the section; if the section pass also through a $\gamma$-tuple generating line $(\gamma \ngtr \beta$, in the same way as for the scroll $S(1,1, m)$ ), then, of the $\beta$ generating lines, $\gamma$ unite together in the $\gamma$-tuple generating line. The general section through a $\gamma$-tuple generating line breaks up into this line counting $\gamma$ times, and a curve of the order $m-\gamma$, which has on

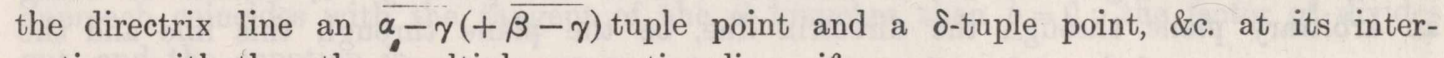
sections with the other multiple generating lines, if any.

\section{Equation of the Scroll $S(1,1, m)$ of the order $m$, Article Nos. 17 and 18.}

17. Taking for the equations of the directrix lines $(x=0, y=0)$ and $(z=0, w=0)$, and supposing that these are respectively an $\alpha$-tuple line and a $\beta$-tuple line on the scroll $\alpha+\beta=m$, it is obvious that the equation of the scroll is

$$
\left(* \gamma(x, y)^{\alpha}(z, w)^{\beta}=0 .\right.
$$

In fact starting 'with this equation, if we consider the section by a plane through the line $(x=0, y=0)$, say the plane $y=\lambda x$, then the equation gives

$$
x^{a}(* \gamma 1, \lambda)^{a}(z, w)^{\beta}=0 ;
$$

that is, the section is made up of the line $(x=0, y=0)$ reckoned $\alpha$ times, and of $\beta$ other lines in the plane $y=\lambda x$; and the like for the section by any plane through the line $(z=0, w=0)$, say the plane $z=\nu w$. Hence the assumed equation represents a scroll of the order $m$, having the two lines for an $\alpha$-tuple line and a $\beta$-tuple line respectively, and conversely such scroll has an equation of the assumed form.

\section{Case of a $\gamma$-tuple generating line.}

18. The multiple generating line meets each of the lines $(x=0, y=0)$ and $(z=0, w=0)$; and we may take for the equations of the multiple generating line $x+y=0, z+w=0$. This being so, the foregoing equation of the scroll may be expressed in the form

or say

$$
(+\gamma x, y)^{\alpha}(z, z+w)^{\beta}=0,
$$

$$
(U, V, W, . .)(z, z+w)^{\beta}=0 \text {, }
$$


where $U, V, W, \ldots$ are functions of the form $(* \gamma x, y)^{\alpha}$. Hence $(\gamma \ngtr \alpha$ or $\beta)$, if the functions $U, V, W, \ldots$ contain respectively the factors $(x+y)^{\gamma},(x+y)^{\gamma-1},(x+y)^{\gamma-2}, \ldots$, the equation will be of the form

$$
(*)(x+y, z+w)^{\gamma}=0
$$

(the coefficients being functions of $x, y, z$ and $z+w$, or, what is the same thing, $x, y, z, w$, of the order $\alpha+\beta-\gamma$ ), and the scroll will therefore have the line $x+y=0, z+w=0$ as a $\gamma$-tuple generating line.

Equation of the Scroll $S(1,1, m)$ of the order m, Article Nos. 19 to 24 .

19. We may take $x=0, y=0$ for the equations of the twofold directrix line, $z=0$ for the equation of the plane of the curve $m$ (an arbitrary plane section of the scroll). Then $(\alpha+\beta=m)$, if the curve $m$ have at the point $(x=0, y=0)$, or foot of the directrix line, an $\alpha(+\beta)$ tuple point, and if moreover we have $y=0$ for the equation of the common tangent of the $\beta$ branches (viz. if the plane $y=0$, instead of being an arbitrary plane through the directrix line, be the plane through this line and the common tangent of the $\beta$ branches), the equation of the curve $m$ will be of the form

$$
\Sigma(y w)^{\beta^{\prime}}\left(* \gamma(x, y)^{a+\beta-2 \beta^{\prime}}=0,\right.
$$

where the summation extends to all integer values of $\beta^{\prime}$ from 0 to $\beta$, both inclusive.

20. Taking $y=\lambda x$ for the equation of any plane through the directrix line, then the corresponding point on the directrix line will be the intersection of this line $(x=0, y=0)$ by the plane $z=\theta w$, where $\theta=\frac{a \lambda+b}{c \lambda+d}$; the foot of the directrix line is given by the value $\theta=0$, or $\lambda=-\frac{b}{a}$, and the equation of the line of approach is therefore $y=-\frac{b}{a} x$; this should coincide with the line $y=0$, which is the common tangent of the $\beta$ branches; that is, we must have $b=0$; I retain, however, for the moment the general value of $b$.

21. The equations of a generating line will be

$$
y=\lambda x, \quad z=\theta w-p x
$$

and then taking $X, Y,(Z=0)$ and $W$ for the coordinates of the point of intersection with the curve $m$, we have

and thence

$$
\begin{aligned}
& Y=\lambda X, \quad 0=\theta W-p X, \\
& \Sigma(Y W)^{\beta^{\prime}}(* \gamma X, Y)^{\alpha+\beta-2 \beta}=0,
\end{aligned}
$$

$$
\Sigma\left(\frac{\lambda p}{\theta}\right)^{\beta^{\prime}}(* \gamma 1, \lambda)^{\alpha+\beta-2 \beta^{\prime}}=0,
$$

or, what is the same thing,

$$
\Sigma \theta^{-\beta^{\prime}}(\lambda p)^{\beta^{\prime}}(* \gamma 1, \lambda)^{\alpha+\beta-2 \beta^{\prime}}=0 ;
$$


which equation, substituting therein for $\theta$ its value in terms of $\lambda$, gives the parameter $p$ which enters into the equations of the generating line; or, what is the same thing, the equation of the scroll is obtained by eliminating $\lambda, \theta, p$ from the equation just mentioned and the equations

$$
y=\lambda x, \quad z=\theta w-p x, \quad \theta=\frac{a \lambda+b}{c \lambda+d} .
$$

22. These last three equations give

$$
\lambda=\frac{y}{x}, \quad \theta=\frac{a y+b x}{c y+d x}, \quad p=\frac{\theta w-z}{x}=\frac{(a y+b x) w-(c y+d x) z}{x} ;
$$

and substituting these values, we find for the equation of the scroll

$$
\Sigma(a y+b x)^{\beta-\beta^{\prime}} y^{\beta^{\prime}}[(a y+b x) w-(c y+d x) z]^{\beta^{\prime}}\left(* \gamma(x, y)^{\alpha+\beta-2 \beta^{\prime}}=0,\right.
$$

which is of the order $a+2 \beta,=2 m-\alpha$, so that the $\alpha(+\beta)$ tuple point, in the case actually under consideration, produces only a reduction $=\alpha$. If however the line of approach coincides with the tangent of the $\beta$ branches, then $b=0$; the factor $y^{\beta}$ divides out, and the equation is

$$
\Sigma(a y w-c y z-d x z)^{\beta^{\prime}}(* \chi \chi x, y)^{a+\beta-2 \beta^{\prime}}=0,
$$

which is of the order $\alpha+\beta,=m$, so that here the reduction caused by the $\alpha(+\beta)$ tuple point is $=\alpha+\beta$. We may without loss of generality substitute $a x$ for $c y+d x$, and then, putting also $a=1$, we find that when the equation of the curve $m$ is as before

$$
\Sigma(y w)^{\beta^{\prime}}\left(* \gamma(x, y)^{\alpha+\beta-2 \beta^{\prime}}=0,\right.
$$

but the plane through the directrix line $(x=0, y=0)$, and the point on this line, are respectively given by the equations $x=\lambda y, z=\lambda w$, the equation of the scroll is

$$
\Sigma(y w-x z)^{\beta^{\prime}}(* \gamma x, y)^{a+\beta-2 \beta^{\prime}}=0 .
$$

23. The result may be verified by considering the section by any plane $y=\lambda x$ through the directrix line. Substituting for $y$ this value, we find

which is of the form

$$
x^{\alpha} \Sigma x^{\beta-\beta^{\prime}}(\lambda w-z)^{\beta^{\prime}}\left(* \gamma(1, \lambda)^{\alpha+\beta-\beta^{\prime}}=0,\right.
$$

$$
x^{a}(+\gamma x, \lambda w-z)^{\beta}=0 ;
$$

so that the section is made up of the directrix line $(x=0, y=0)$ reckoned $\alpha$ times and of $\beta$ lines in the plane $y-\lambda x=0$, the intersections of the plane $y-\lambda x=0$ by planes such as $z=\lambda w-p x$.

Case of a $\gamma$-tuple generating line.

24. The equation of the scroll may be written

$$
(U, V, W, \ldots \curlyvee 1, y w-x z)^{\beta}=0,
$$

C. V. 
where $U, V, W, \ldots$ are functions of $x, y$ of the forms

$$
(* \chi x, y)^{m}, \quad(* \chi x, y)^{m-2}, \quad(* \chi x, y)^{m-4}, \ldots ;
$$

assuming that these contain respectively the factors

$$
(y-\kappa x)^{\gamma}, \quad(y-\kappa x)^{\gamma-1}, \quad(y-\kappa x)^{\gamma-2} \ldots,
$$

where $\gamma \ngtr \frac{1}{2} m$, then the equation takes the form

$$
\left(U^{\prime}, V^{\prime}, W^{\prime} \ldots \gamma y-\kappa x, w(y-\kappa x)+x(\kappa w-z)\right)^{\gamma}=0,
$$

where the coefficients $U^{\prime}, V^{\prime}, W^{\prime}, \ldots$ are functions of $x, y, z, w$ of the orders $m-\gamma$, $m-\gamma-1, m-\gamma-2, \ldots ;$ or, what is the same thing, the equation is

$$
\left(U^{\prime \prime}, V^{\prime \prime}, W^{\prime \prime}, \ldots \nmid y-\kappa x, \kappa w-z\right)^{\gamma}=0,
$$

where $U^{\prime \prime}, V^{\prime \prime}, W^{\prime \prime}, \ldots$ are functions of $x, y, z, w$ of the order $m-\gamma$. The scroll has thus the $\gamma$-tuple generating line

$$
y-\kappa x=0, \kappa w-z=0 .
$$

Cubic Scrolls, Article Nos. 25 to 35 .

25. In the case of a cubic scroll there is necessarily a nodal( $\left.{ }^{1}\right)$ line; in fact for the $m$-thic scroll there is a nodal curve which is of the order $m-2$ at least, and of the order $\frac{1}{2}(m-1)(m-2)$ at most, and which for $m=3$ is therefore a right line. And moreover we see at once that every cubic surface having a nodal line is a scroll; in fact any plane whatever through the nodal line meets the surface in this line counting as 2 lines, and in a curve of the order 1 ; that is, a line; there are consequently on the surface an infinity of lines, or the surface is a scroll. We have therefore to examine the cubic surfaces which have a nodal line.

26. Let the equations of the nodal line be $x=0, y=0$; then the equation of the surface is

$$
U z+V w+Q=0,
$$

where $U, V, Q$ are functions of $(x, y)$ of the orders 2, 2, 3 respectively. Suppose first that $U, V$ have no common factor, then we may write

$$
Q=(\alpha x+\beta y) U+(\gamma x+\delta y) V
$$

and substituting this value, and changing the values of $z$ and $w$, the equation of the surface is of the form

or, what is the same thing,

$$
U z+V w=0,
$$

$$
\left(* \gamma(x, y)^{2}(z, w)=0\right. \text {; }
$$

1 The nodal line of a cubic scroll is of course a double line, and in regard to these scrolls the epithets 'nodal' and 'double' may be used indifferently. 
so that, besides the nodal directrix line $(x=0, y=0)$, the scroll has the simple directrix line $(z=0, w=0)$ : it is clear that the section by any plane whatever is a cubic curve having a node at the foot of the nodal directrix line $(x=0, y=0)$, and passing through the foot of the simple directrix line $(z=0, w=0)$; that is, it is a cubic scroll of the kind $S(1,1,3)$; and since for $m=3$ the only partition $m=\alpha+\beta$ is $m=2+1$, there is only one kind of cubic scroll $S(1,1,3)$, and we may say simpliciter that the scroll in question is the cubic scroll $S(1,1,3)$.

27. If however the functions $U, V$ have a common factor, say $(\lambda x+\mu y)$, then $z U+w V$ will contain this same factor, and the remaining factor will be of the form

$$
z(\alpha x+\beta y)+w(\gamma x+\delta y), \quad=y(\beta z+\delta w)+x(\alpha z+\gamma w),
$$

or, changing the values of $z$ and $w$, the remaining factor will be of the form $y w-x z$, and the equation of the scroll thus is

$$
(\lambda x+\mu y)(y w-x z)+\left(* \gamma(x, y)^{3}=0,\right.
$$

where it is clear that the section by any plane whatever is a cubic curve having a node at the foot of the directrix line $x=0, y=0$. The scroll is thus a cubic scroll of the form $S(\overline{1,1}, 3)$, viz. it is the scroll of the kind where the section is a cubic curve with a $2(+1)$ tuple point (ordinary double point, or node), the line of approach being one of the two tangents at the node; and since for $m=3$ the only partition $m=\alpha+\beta$ is $m=2+1$, there is only one kind of cubic scroll $S(\overline{1,1}, 3)$, and we may say simpliciter that the scroll in question is the cubic scroll $S(\overline{1,1}, 3)$. The conclusion therefore is that for cubic scrolls we have only the two kinds, $S(1,1,3)$ and $S(\overline{1,1}, 3)$. The foregoing equations of these scrolls admit however of simplification; and I will further consider the two kinds respectively.

\section{The Cubic Scroll S(1, 1, 3).}

28. Starting from the equation

$$
(* \chi x, y)^{2}(z, w)=0 \text {, }
$$

or, writing it at full length,

$$
z\left(a, b, c \gamma(x, y)^{2}+w\left(a^{\prime}, b^{\prime}, c^{\prime} \chi x, y\right)^{2}=0,\right.
$$

we may find $\theta_{1}, \theta_{2}$ so that

$$
\begin{aligned}
& (a, b, c \chi x, y)^{2}+\theta_{1}\left(a^{\prime}, b^{\prime}, c^{\prime} \chi x, y\right)^{2}=\left(p_{1} x+q_{1} y\right)^{2}, \\
& \left(a, b, c \chi(x, y)^{2}+\theta_{2}\left(a^{\prime}, b^{\prime}, c^{\prime} \chi x, y\right)^{2}=\left(p_{2} x+q_{2} y\right)^{2},\right.
\end{aligned}
$$

$\theta_{1}$ and $\theta_{2}$ being unequal, since by hypothesis $(a, b, c \chi x, y)^{2}$ and $\left(a^{\prime}, b^{\prime}, c^{\prime} \gamma x, y\right)^{2}$ have no common factor. This gives

$$
\begin{aligned}
& (a, b, c \gamma x, y)^{2}=\alpha\left(p_{1} x+q_{1} y\right)^{2}+\beta\left(p_{2} x+q_{2} y\right)^{2} \\
& \left(a^{\prime}, b^{\prime}, c^{\prime} \chi x, y\right)^{2}=\gamma\left(p_{1} x+q_{1} y\right)^{2}+\delta\left(p_{2} x+q_{2} y\right)^{2}
\end{aligned}
$$

or the equation becomes

$$
(\alpha z+\gamma w)\left(p_{1} x+q_{1} y\right)^{2}+(\beta z+\delta w)\left(p_{2} x+q_{2} y\right)^{2}=0 ;
$$


or changing the values of $(x, y)$ and of $(z, w)$, the equation is

$$
x^{2} z+y^{2} w=0,
$$

which may be considered as the canonical form of the equation. It may be noticed that the Hessian of the form is $x^{2} y^{2}$.

29. We may of course establish the theory of the surface from the equation $x^{2} z+y^{2} w=0$; the equation is satisfied by $x=\lambda y, w=-\lambda^{2} z$, which are the equations of a line meeting the line $(x=0, y=0)(1)$ and the line $(z=0, w=0)\left(1^{\prime}\right)$. The generating line meets also any plane section of the surface; in fact, if the equation of the plane of the section be $\alpha x+\beta y+\gamma z+\delta w=0$, then we have at once

$$
x: y: z: w=\delta \lambda^{3}-\gamma \lambda: \delta \lambda^{2}-\gamma: \alpha \lambda+\beta:-\alpha \lambda^{3}-\beta \lambda^{2}
$$

for the coordinates of the point of intersection.

30. The form of the equation shows that there are on the line 1 two points, viz. the points $(x=0, y=0, z=0)$ and $(x=0, y=0, w=0)$, through each of which there passes a pair of coincident generating lines: calling these $A$ and $B$, then, if the coincident lines through $A$ meet the line $1^{\prime}$ in $C$, and the coincident lines through $B$ meet the line $1^{\prime}$ in $D$, it is easy to see that $x=0, y=0, z=0$, and $w=0$ will denote the equations of the planes $B A C, B A D, B C D$, and $A C D$ respectively.

31. We obtain also the following construction: take a cubic curve having a node, and from any point $K$ on the curve draw to the curve the tangents $K p, K q$; through the points of contact draw at pleasure the lines $p A C$ and $q B D$; through the node draw a line meeting these two lines in the points $A, B$ respectively, this will be the line 1; and through the point $K$ a line meeting the same two lines in the points $C$ and $D$ respectively, this will be the line $1^{\prime}$; and, the equations $x=0, y=0, z=0, w=0$ denoting as above, the equation of the surface will be $x^{2} z+y^{2} w=0$.

The points $A$ and $B$ are cuspidal points on the nodal line; any section of the scroll by a plane through one of these points is a cubic curve having at the point in question a cusp.

32. It is to be noticed however that the cuspidal points are not of necessity real ; if for $x, y$ we write $x+\iota y, x-\imath y$, and in like manner $z+\iota w, z-\iota w$ for $z$, w, then the equation takes the form

$$
\left(x^{2}-y^{2}\right) z-2 x y w=0,
$$

which is a cubic scroll $S(1,1,3)$ with the cuspidal points imaginary.

In the last-mentioned case the nodal line is throughout its whole length crunodal; in the case first considered, where the equation is $x^{2} z+y^{2} w=0$, the nodal line is for that part of its length for which $z, w$ have opposite signs, crunodal; and for the remainder of its length, or where $z, w$ have the same sign, acnodal. There are two different forms, according as the line is for the portion intermediate between the cuspidal points crunodal and for the extramediate portions acnodal, or as it is for the intermediate portion acnodal and for the extramediate portions crunodal. 


\section{Cubic Scroll S(1, 1, 3).}

33. Starting from the equation

$$
(\lambda x+\mu y)(y w-x z)+(* \gamma x, y)^{3}=0,
$$

then putting $w-\mu z$ for $w$ and $\lambda z$ for $z$, this may be written

$$
(\lambda x+\mu y)\{y w-z(\lambda x+\mu y)\}+(* \gamma \lambda x+\mu y, y)^{3}=0,
$$

or, what is the same thing,

$$
x(y w-x z)+(* \gamma x, y)^{3}=0
$$

and then, if $\left(* \gamma(x, y)^{3}=\left(\alpha, \beta, \gamma, \delta^{\gamma}(x, y)^{3}\right.\right.$, this may be written

$$
x\{y(w+\beta x+\gamma y)-x(z-\alpha x)\}+\delta y^{3}=0 ;
$$

or changing the values of $w$ and $z$, we have

$$
x(y w-x z)+y^{3}=0
$$

for the equation of the scroll $S(\overline{1,1}, 3)\left({ }^{1}\right)$.

34. The Hessian of the form is $x^{4}$, and it thus appears that the plane $x=0$ is a determinate plane through the double line. But $y=0$ is not a determinate plane; in fact, if for $y$ we write $y+\lambda x$, the equation is

that is

$$
-x^{2} z+x w(y+\lambda x)+(y+\lambda x)^{3}=0,
$$

$$
-x^{2}\left(z-\lambda w-3 \lambda^{2} y-\lambda^{3} x\right)+x y(w+3 \lambda x)+y^{3}=0,
$$

which, changing $z$ and $w$, is still of the form $x(y w-x z)+y^{3}=0$.

The planes $z=0, w=0$ will alter with the plane $y=0$, but they are not determined even when the plane $y=0$ is determined; in fact we may, without altering the equation, change $w, z$ into $w+\theta y, z+\theta x$ respectively.

35. In the equation $x(y w-x z)+y^{3}=0$, writing $y=\lambda x$, we find for the equations of a generating line, $y=\lambda x, z=\lambda w+\lambda^{3} x$. Considering the section by the plane $\alpha x+\beta y+\gamma z+\delta w=0$, we have

$$
x: y: z: w=-\gamma \lambda-\delta:-\gamma \lambda^{2}-\delta \lambda:-\delta \lambda^{3}+\beta \lambda^{2}+\alpha \lambda: \gamma \lambda^{3}+\beta \lambda+\alpha
$$

for the coordinates of the point where the generating line meets the section.

The generating line meets the nodal line at the intersection of the nodal line by the plane $z=\lambda w$; that is, the points $z=\lambda w$ on the nodal line correspond to the planes $y=\lambda x$ through the nodal line. In particular the point $w=0$ on the nodal line corresponds to the plane $x=0$ through the nodal line: the point $\gamma z+\delta w=0$ on the nodal line (that is, the point where this line is met by the plane $\alpha x+\beta y+\gamma z+\delta w=0$ ) corresponds to the plane $\gamma x+\delta y=0$ through the nodal line; the intersections of the plane $\alpha x+\beta y+\gamma z+\delta w=0$ by this plane $\gamma x+\delta y=0$, and by the plane $x=0$, are the tangents of the section at the node. form.

${ }^{1}$ It is somewhat more convenient to change the sign of $z$, and take $x(y z v+x z)+y^{3}=0$ as the canonical 
Quartic Scrolls, Article Nos. 36 to 50.

36. We may consider, first, the quartic scrolls $S(1,1,4)$. The section is a quartic curve having an $\alpha$-tuple point and a $\beta$-tuple point, where $\alpha+\beta=4$; that is, we have $\alpha=2, \beta=2$, a quartic with two nodes (double points), or else $\alpha=3, \beta=1$, a quartic with a triple point. But the case $\alpha=2, \beta=2$ gives rise to two species: viz., in general the quartic has only the two double points, and we have then a scroll with two nodal (2-tuple) directrix lines, and without any nodal generator; the section may however have a third double point, and the scroll has then a nodal (double) generator. For the case $\alpha=3, \beta=1$, the section admits of no further singularity, and we have a quartic scroll with a triple directrix line and a single directrix line.

37. Next for the quartic scroll $S(1,1,4)$. The section is here a quartic curve with an $\alpha(+\beta)$ tuple point, where $\alpha+\beta=4$; that is, $\alpha=2, \beta=2$, or else $\alpha=3, \beta=1$. In the former case the section has a $2(+2)$ tuple point, that is, a double point where the two branches have a common tangent-otherwise, two coincident double points: say the curve has a tacnode; the line of approach is the tangent at the tacnode. We have here a scroll with a twofold double line; there are however two cases: viz., in general the section has, besides the tacnode, no other double point; that is, the scroll has no nodal generator: the section may however have a third double point, and the scroll has then a nodal (double) generator. In the case $\alpha=3, \beta=1$ the section has a triple point, and the line of approach is the tangent at one of the branches at the triple point; the scroll has a twofold, say a $3(+1)$ tuple directrix line: as the section admits of no further singularity, this is the only case. The foregoing enumeration gives three species of quartic scrolls $S(1,1,4)$, and three species of quartic scrolls $S(\overline{1,1}, 4)$, together six species, viz. these are as follows :

\section{Quartic Scroll, First Species, $S\left(1_{2}, 1_{2}, 4\right)$, with two double directrix lines, and without a nodal generator.}

38. Taking $(x=0, y=0)$ and $(z=0, w=0)$ for the equations of the two directrix lines respectively, the equation of the scroll is

$$
(* \gamma x, y)^{2}(z, w)^{2}=0 \text {. }
$$

\section{Quartic Scroll, Second Species, $S^{\prime}\left(1_{2}, 1_{2}, 4\right)$, with two double directrix lines and with a double generator.}

39. This is in fact a specialized form of the first species, the difference being that there is a nodal (double) generator. Supposing as before that the equations of the directrix lines are $(x=0, y=0)$ and $(z=0, w=0)$ respectively; let the equations of the nodal generator be $(x+y=0, z+w=0)$; then, observing that for the first species the equation may be written $(* \gamma x, y)^{2}(z, z+w)^{2}=0$, it is clear that if the terms in $z^{2}$ and $z(z+w)$ are divisible by $(x+y)^{2}$ and $(x+y)$ respectively, the surface will have as a new 
double line the line $(x+y=0, z+w=0)$, which will be a double generator; and we thus arrive at the equation of the second species of quartic scrolls, viz. this is

$$
\left((x+y)^{2},(x+y)(x, y),(x, y)^{2} \gamma z, z+w\right)^{2}=0 .
$$

Quartic Scroll, Third Species, $S\left(1_{3}, 1,4\right)$, with a triple directrix line and a single directrix line.

40. Taking $(x=0, y=0)$ for the equations of the triple directrix line, and $(z=0, w=0)$ for the equations of the single directrix line, the equation is

$$
\left(* \gamma(x, y)^{3}(z, w)=0 .\right.
$$

Quartic Scroll, Fourth Species, $S\left(\overline{1}_{2}, 1_{2}, 4\right)$, with a twofold $(2(+2)$ tuple $)$ directrix line, and without a nodal generator.

41. Taking $(x=0, y=0)$ for the equations of the directrix line, $z=0$ for that of a plane section of the scroll, $y=0$ for the equation of a plane through the tangent at the tacnode of the section, and supposing (see ante, No. 22) that the plane through the directrix line and the corresponding point on this line are respectively given by the equations $x=\lambda y$ and $z=\lambda w$, the equation of the scroll is

$$
(y w-x z)^{2}+(y w-x z)(x, y)^{2}+(x, y)^{4}=0 .
$$

Quartic Scroll, Fifth Species, $S^{\prime \prime}\left({\overline{1_{2},}}_{1}, 4\right)$, with a twofold $(2(+2)$ tuple) generating line, and with a double generator.

42. Let the equations of the double generator be $x+y=0, z+w=0$; then the line in question must be a double line on the surface represented by the lastmentioned equation, and this will be the case if only the second and third terms contain the factors $(x+y)$ and $(x+y)^{2}$ respectively. The equation for the fifth species consequently is

$$
(y w-x z)^{2}+2(y w-x z)(x+y)(x, y)+(x+y)^{2}(x, y)^{2}=0 .
$$

Quartic Scroll, Sixth Species, $S\left(\overline{1_{3}, 1}, 4\right)$, with a twofold $(3(+1)$ tuple) generating line.

43. Taking $(x=0, y=0)$ 'for the equations of the directrix line, $z=0$ for the equation of a plane section, and assuming that the plane $y=0$ passes through the tangent which is the line of approach, and that the plane through the directrix line and the corresponding point on this line are respectively given by the equations $x=\lambda y$ and $z=\lambda w$, the equation of the scroll is

$$
(y w-x z)(x, y)^{2}+(x, y)^{4}=0 .
$$


I refrain on the present occasion from a more particular discussion of the foregoing six species of quartic scrolls. I establish two other species, as follows:

Quartic Scroll, Seventh Species, S(1, 2, 2), with nodal directrix line, and nodal directrix conic which meet, and with a simple directrix conic which meets the nodal conic in two points.

44. We see, à priori, that the scroll generated as above will be of the order 4, that is, a quartic scroll. In fact using the formula (ante, No. o)),

we have here

$$
\text { Order }=2 m n p-\alpha m-\beta n-\gamma p,
$$

Nodal conic, $m=2, \quad \alpha=0$,

Simple conic, $n=2, \quad \beta=1$,

and hence

$$
\text { Line } \quad, p=1, \gamma=2 \text {, }
$$

$$
\text { Order }=8-2-2,=4 \text {. }
$$

45. Take $(x=0, y=0)$ for the equations of the directrix line, $z=0$ for the equation of the plane of the simple conic, $w=0$ for that of the plane of the nodal conic; since the conics intersect in two points, they lie on a quadric surface, say the surface $U=0$; the equations of the simple conic thus are $z=0, U=0$; those of the nodal conic are $w=0, U=0$. The directrix line $x=0, y=0$ meets the nodal conic; that is, $U$ must vanish identically for $x=0, y=0, w=0$; and this will be the case if only the term in $z^{2}$ is wanting; that is, we must have

$$
U=\left(a, b, 0, d, f, g, h, l, m, n \gamma(x, y, z, w)^{2} .\right.
$$

But we may in the first instance omit the condition in question, and write

$$
U=(a, b, c, d, f, g, h, l, m, n \gamma x, y, z, w)^{2} ;
$$

this would lead to a sextic instead of a quartic scroll.

46. The equations of a generating line (since it meets the directrix line $x=0, y=0$ ) may be taken to be

$$
x=\alpha y, \quad z=\beta\left(y-\frac{w}{\theta}\right) ;
$$

the condition in order to the intersection of the generating line with the nodal conic is at once found to be

$$
a \alpha^{2}+2 h \alpha+b+2 \beta(f+g \alpha)+c \beta^{2}=0,
$$

and that for its intersection with the simple conic

$$
a \alpha^{2}+2 h \alpha+b+2 \theta(m+l \alpha)+d \theta^{2}=0 ;
$$

and writing the equations of the generating line in the form

$$
\alpha=\frac{y}{x}, \quad \theta=\frac{\beta w}{\beta y-z},
$$


the elimination of $\alpha, \beta, \theta$ from these four equations gives the required equation of the scroll. Writing for a moment

we find

$$
\begin{aligned}
& \Theta=a \alpha^{2}+2 h \alpha+\beta \\
& F=g \alpha+f \\
& M=l \alpha+m
\end{aligned}
$$

$$
\begin{aligned}
& c \beta^{2}+2 F \beta+\Theta=0, \\
& \left(\Theta y^{2}+2 M y w+d w^{2}\right) \beta^{2}-2(\Theta y z+M w z) \beta+\Theta z^{2}=0 ;
\end{aligned}
$$

or, introducing at this place the condition $c=0$, the first equation gives $\beta$ linearly, and we thence obtain

$$
\Theta\left(\Theta y^{2}+2 M y w+d w^{2}\right)+4 F(\Theta y z+M w z)+4 F^{2} z^{2}=0,
$$

or, what is the same thing,

$$
(\Theta y+2 F z)^{2}+2 M w(\Theta y+2 F z)+\Theta d w^{2}=0 ;
$$

whence, observing that we have

$$
\Theta=\frac{a x^{2}+2 h x y+b y^{2}}{y^{2}}, \quad F=\frac{g x+f y}{y}, \quad M=\frac{l x+m y}{y},
$$

the equation of the scroll is

$$
\begin{aligned}
& \left(a x^{2}+2 h x y+b y^{2}+2 g z x+2 f y z\right)^{2} \\
+ & 2\left(a x^{2}+2 h x y+b y^{2}+2 g z x+2 f y z\right)(l x+m y) w \\
+ & \left(a x^{2}+2 h x y+b y^{2}\right) d w^{2}=0 .
\end{aligned}
$$

We see from the equation that the surface contains the line $(x=0, y=0)$ as a double line, the conic

$$
w=0, \quad a x^{2}+2 h x y+b y^{2}+2 g z x+2 f y z=0
$$

as a double curve, also the conic

$$
z=0, \quad a x^{2}+2 h x y+b y^{2}+2 l x w+2 m y w+d w^{2}=0
$$

as a simple curve on the surface, the complete intersection by the plane $z=0$ being in fact the last-mentioned conic, and the pair of lines

$$
z=0, \quad a x^{2}+2 h x y+b y^{2}=0 .
$$

Quartic Scroll, Eighth Species, S(1, 3), with a directrix line, and a directrix skew cubic met twice by each generating line.

47. We see, a priori, that the scroll is of the order 4, that is, a quartic scroll; in fact for the quartic scroll $S\left(1, m^{2}\right)$ the order is $=[m]^{2}+M$ (first memoir, p. 457 [ante p. 172]), and we have here $m=3, M=h-\frac{1}{2}[m]^{2}=1-3=-2$; that is, order $=6-2,=4$.

c. $\mathbf{v}$. 
48. The equations of the cubic curve may be taken to be

$$
\left|\begin{array}{lll}
x, & y, & z \\
y, & z, & w
\end{array}\right|=0
$$

or, what is the same thing,

$$
x z-y^{2}=0, \quad x w-y z=0, \quad y w-z^{2}=0 ;
$$

those of the directrix line may be represented by

$$
\begin{aligned}
& \alpha x+\beta y+\gamma z+\delta w=0, \\
& \alpha^{\prime} x+\beta^{\prime} y+\gamma^{\prime} z+\delta^{\prime} w=0 ;
\end{aligned}
$$

or, what is the same thing, if

$$
\begin{array}{ll}
\beta \gamma^{\prime}-\beta^{\prime} \gamma=a, & \alpha \delta^{\prime}-\alpha^{\prime} \delta=f \\
\gamma \alpha^{\prime}-\gamma^{\prime} \alpha=b, & \beta \delta^{\prime}-\beta^{\prime} \delta=g, \\
\alpha \beta^{\prime}-\alpha^{\prime} \beta=c, & \gamma \delta^{\prime}-\gamma^{\prime} \delta=h
\end{array}
$$

(and therefore identically $a f+b g+c h=0$ ), the line is defined by means of its "six coordinates" $(a, b, c, f, g, h)$.

49. The equations of the cubic curve are satisfied by writing therein

$$
x: y: z: w=1: t: t^{2}: t^{3},
$$

and therefore the coordinates of any two points on the curve may be represented by $\left(1, \theta, \theta^{2}, \theta^{3}\right)$ and $\left(1, \phi, \phi^{2}, \phi^{3}\right)$; hence, if $x, y, z, w$ are the coordinates of a point in the line joining the last mentioned two points, we have

$$
x: y: z: w=l+m: l \theta+m \phi: l \theta^{2}+m \phi^{2}: l \theta^{3}+m \phi^{3},
$$

which equations, treating therein $l, m$ as indeterminate parameters, give the equations of the line in question. And putting moreover

we have identically

$$
p=y w-z^{2}, \quad q=y z-x w, \quad r=x z-y^{2},
$$

$$
p: q: r=\theta \phi:-(\theta+\phi): 1 .
$$

50. In order that the line in question may meet the directrix line, we must have

$$
\begin{aligned}
& l\left(\alpha+\beta \theta+\gamma \theta^{2}+\delta \theta^{3}\right)+m\left(\alpha+\beta \phi+\gamma \phi^{2}+\delta \phi^{3}\right)=0, \\
& l\left(\alpha^{\prime}+\beta^{\prime} \theta+\gamma^{\prime} \theta^{2}+\delta^{\prime} \theta^{3}\right)+m\left(\alpha^{\prime}+\beta^{\prime} \phi+\gamma^{\prime} \phi^{2}+\delta^{\prime} \phi^{3}\right)=0 ;
\end{aligned}
$$

that is, eliminating $l$ and $m$, we must have

$$
\left|\begin{array}{cc}
\alpha+\beta \theta+\gamma \theta^{2}+\delta \theta^{3}, & \alpha+\beta \phi+\gamma \phi^{2}+\delta \phi^{3} \\
\alpha^{\prime}+\beta^{\prime} \theta+\gamma^{\prime} \theta^{2}+\delta^{\prime} \theta^{3}, & \alpha^{\prime}+\beta^{\prime} \phi+\gamma^{\prime} \phi^{2}+\delta^{\prime} \phi^{3}
\end{array}\right|=0,
$$


or, developing,

$$
\begin{aligned}
& \left(\alpha \beta^{\prime}-\alpha^{\prime} \beta\right)(\phi-\theta)+\left(\alpha \gamma^{\prime}-\alpha^{\prime} \gamma\right)\left(\phi^{2}-\theta^{2}\right)+\left(\alpha \delta^{\prime}-\alpha^{\prime} \delta\right)\left(\phi^{3}-\theta^{3}\right) \\
& \quad+\left(\beta \gamma^{\prime}-\beta^{\prime} \gamma\right)\left(\theta \phi^{2}-\theta^{2} \phi\right)+\left(\beta \delta^{\prime}-\beta^{\prime} \delta\right)\left(\theta \phi^{3}-\theta^{3} \phi\right)+\left(\gamma \delta^{\prime}-\gamma^{\prime} \delta\right)\left(\theta^{2} \phi^{3}-\theta^{3} \phi^{2}\right)=0
\end{aligned}
$$

the several terms in $(\theta, \phi)$, each divided by $\phi-\theta$, give respectively

$$
1, \phi+\theta,(\phi+\theta)^{2}-\phi \theta, \theta \phi, \theta \phi(\phi+\theta), \theta^{2} \phi^{2},
$$

which are equal to

$$
\left(r^{2},-q r, \quad q^{2}-p r \quad, p r, \quad-p q \quad, \quad p^{2}\right) \text {; }
$$

hence replacing also $\alpha \beta^{\prime}-\alpha^{\prime} \beta$, \&c. by their values $c$, \&c., we find

$$
(c,-b, f, a, g, h)\left(r^{2},-q r, q^{2}-p r, p r,-p q, p^{2}\right)=0,
$$

or, what is the same thing,

$$
\left(h, f, c, b, a-f,-g^{\gamma} \gamma p, q, r\right)^{2}=0,
$$

where the coefficients $(a, b, c, f, g, h)$ satisfy the relation $a f+b g+c h=0 ; p, q, r$ stand respectively for $y w-z^{2}, y z-x w, x z-y^{2}$.

Writing for greater convenience

$$
(h, f, c, b, a-f,-g)=(a, b, c, 2 f, 2 g, 2 h),
$$

or, what is the same thing,

then we have

$$
(a, b, c, f, g, h) \quad=(\mathrm{b}+2 \mathrm{~g}, 2 \mathrm{f}, \mathrm{c}, \mathrm{b},-2 \mathrm{~h}, \mathrm{a})
$$

$$
a f+b g+c h=a c+b^{2}+2 b g-4 f h=0 ;
$$

and hence finally we have for the equation of the scroll $S\left(1,3^{2}\right)$,

$$
\left(\mathrm{a}, \mathrm{b}, \mathrm{c}, \mathrm{f}, \mathrm{g}, \mathrm{h} \gamma y w-z^{2}, \quad y z-x w, \quad x z-y^{2}\right)^{2}=0,
$$

where the coefficients satisfy the relation

$$
a c+b^{2}+2 b g-4 f h=0 .
$$

The equations of the directrix cubic are of course

$$
y w-z^{2}=0, \quad y z-x w=0, \quad x z-y^{2}=0 ;
$$

and the directrix line is given by its six coordinates,

$$
(b+2 g, 2 f, c, b,-2 h, a) \text {. }
$$

On the general Theory of Scrolls, Article Nos. 51 to 53.

51. I annex in conclusion the following considerations on the general theory of scrolls. Consider a scroll of the $n$th order; the intersection by an arbitrary plane, say the plane $w=0$, is a curve of the $n$th order $(* \chi x, y, x)^{n}=0$; any point $(x, y, z, 0)$ 
where $(x, y, z)$ satisfy the foregoing equation, is the foot of a generating line; and we may imagine this generating line determined by means of the coordinates $(X, Y, Z, W)$, given functions of $(x, y, z)$ of a point on the line. This being so, the "six coordinates," say $(p, q, r, s, t, u)$, of the line are

viz.

$$
\left\|\begin{array}{llll}
X, & Y, & Z, & W \\
x, & y, & z, & 0
\end{array}\right\|
$$

$$
\begin{array}{ll}
p=Y z-Z y, & s=-W x \\
q=Z x-X z, & t=-W y, \\
r=X y-Y x, & u=-W z
\end{array}
$$

or, writing for greater convenience $-v$ in the place of $W$, the six coordinates of the line are $p, q, r, v x, v y, v z$, where $p, q, r$ are functions of $(x, y, z)$, connected by the relation $p x+q y+v z=0$; and $v$ is also a function of $(x, y, z)$.

52. Consider the intersection of the surface by an arbitrary line, the six coordinates whereof are $(A, B, C, F, G, H)$; then for the generating lines which meet this line we have

$$
v(A x+B y+C z)+F p+G q+H r=0,
$$

and this equation, together with the equation $(* \gamma x, y, z)^{n}=0$, determines $(x, y, z)$, the coordinates of the foot of a generating line which meets the arbitrary line $(A, B, C, F, G, H)$. Since the order of the scroll is $=n$, the number of such generating lines should be $=n$, that is, there should be $n$ relevant intersections of the two curves,

$$
\begin{aligned}
& v(A x+B y+C z)+F p+G q+H r=0, \\
& (* \gamma x, y, z)^{n}=0 ;
\end{aligned}
$$

but if $(p, q, r, v x, v y, v z)$ are each of the order $k$, the number of actual intersections is $=k n$, which is too many by $(k-1) n$.

53. Suppose that the curves

or say the curves

$$
p=0, \quad q=0, \quad r=0, \quad v x=0, \quad v y=0, \quad v z=0,
$$

$$
p=0, \quad q=0, \quad r=0, \quad v=0
$$

have in common $\theta$ intersections, and let these be points of the multiplicities $\alpha_{1}, \alpha_{2}, \alpha_{3}, \ldots \alpha_{\theta}$ on the curve $\left(* \gamma(x, y, z)^{n}=0\right.$ (viz. according as the curve does not pass through any one of the intersections in question, or passes once, twice, \&c. through such intersection, we have for that intersection $\alpha_{1}=0,1,2$, \&c., as the case may be, and so for the other intersections); then the $k n$ points of intersection include the $\alpha_{1}+\alpha_{2} \ldots+\alpha_{\theta}$, or say the $\Sigma \alpha$ intersections; but these, being independent of the line $(A, B, C, F, G, H)$ under consideration, are irrelevant points, and the number of relevant points of intersection is $k n-\Sigma \alpha$; that is, if we have $\Sigma \alpha=(k-1) n$, then the scroll in question, viz. the scroll generated by a line which meets the plane $w=0$ in the curve $(* \gamma x, y, z)^{n}=0$, and which has for its six coordinates $(p, q, r, v x, v y, v z)$, will be a scroll of the $n$th order. 